\title{
Detection of antibody responses against Streptococcus pneumoniae, Haemophilus influenzae, and Moraxella catarhalis proteins in children with community-acquired pneumonia: effects of combining pneumococcal antigens, pre-existing antibody levels, sampling interval, age, and duration of illness
}

\author{
I. C. Borges ${ }^{1}$ • D. C. Andrade ${ }^{1}$ - A.-L. Vilas-Boas ${ }^{1}$ - M.-S. H. Fontoura ${ }^{2} \cdot$ H. Laitinen $^{3}$ \\ N. Ekström ${ }^{3}$ - P. V. Adrian ${ }^{4}$ - A. Meinke ${ }^{5}$ - M.-R. A. Cardoso ${ }^{6}$ - A. Barral ${ }^{1,7}$. \\ O. Ruuskanen ${ }^{8}$ - H. Käyhty ${ }^{3}$ - C. M. Nascimento-Carvalho ${ }^{1,2}$
}

Received: 27 March 2015 / Accepted: 31 March 2015 /Published online: 19 April 2015

(C) Springer-Verlag Berlin Heidelberg 2015

\begin{abstract}
We evaluated the effects of combining different numbers of pneumococcal antigens, pre-existing antibody levels, sampling interval, age, and duration of illness on the detection of IgG responses against eight Streptococcus pneumoniae proteins, three Haemophilus influenzae proteins, and five Moraxella catarrhalis proteins in 690 children aged $<5$ years with pneumonia. Serological tests were performed on acute and convalescent serum samples with a multiplexed bead-based immunoassay. The median sampling interval was
\end{abstract}

\section{C. Borges}

igorcms@gmail.com

1 Postgraduate Programme in Health Sciences, Federal University of Bahia School of Medicine, Praça XV de Novembro, s/n - Largo do Terreiro de Jesus, Salvador, Bahia 40025-010, Brazil

2 Department of Paediatrics, Federal University of Bahia School of Medicine, Salvador, Bahia, Brazil

3 National Institute for Health and Welfare, Helsinki, Finland

4 DST/NRF Vaccine Preventable Diseases, MRC Respiratory and Meningeal Pathogens Research Unit, University of the Witwatersrand, Johannesburg, South Africa

5 Valneva Austria GmbH, Campus Vienna Biocenter 3, Vienna, Austria

6 Department of Epidemiology, University of São Paulo School of Public Health, São Paulo, Brazil

7 Department of Pathology, Federal University of Bahia School of Medicine and Centro de Pesquisas Gonçalo Moniz, Fundação Oswaldo Cruz, Salvador, Bahia, Brazil

8 Department of Paediatrics, Turku University and University Hospital, Turku, Finland
19 days, the median age was 26.7 months, and the median duration of illness was 5 days. The rate of antibody responses was $15.4 \%$ for at least one pneumococcal antigen, $5.8 \%$ for $H$. influenzae, and $2.3 \%$ for M. catarrhalis. The rate of antibody responses against each pneumococcal antigen varied from 3.5 to $7.1 \%$. By multivariate analysis, pre-existing antibody levels showed a negative association with the detection of antibody responses against pneumococcal and $H$. influenzae antigens; the sampling interval was positively associated with the detection of antibody responses against pneumococcal and $H$. influenzae antigens. A sampling interval of 3 weeks was the optimal cut-off for the detection of antibody responses against pneumococcal and $H$. influenzae proteins. Duration of illness was negatively associated with antibody responses against PspA. Age did not influence antibody responses against the investigated antigens. In conclusion, serological assays using combinations of different pneumococcal proteins detect a higher rate of antibody responses against S. pneumoniae compared to assays using a single pneumococcal protein. Pre-existing antibody levels and sampling interval influence the detection of antibody responses against pneumococcal and $H$. influenzae proteins. These factors should be considered when determining pneumonia etiology by serological methods in children.

\section{Introduction}

Community-acquired pneumonia (CAP) represents an important burden for childhood healthcare throughout the world [1]. In order to reach CAP control, it is important to understand the 
relative importance of etiological agents for the implementation of treatment and preventive strategies. However, it is difficult to identify CAP causative agents. Blood culture is a poorly sensitive diagnostic tool, as it detects Streptococcus pneumoniae bacteremia in less than $5 \%$ of pediatric CAP cases. Therefore, epidemiological studies have relied mostly on the use of serological techniques to identify microbespecific antibodies. The use of such a diagnostic strategy is not completely validated, mainly because of the lack of a gold standard diagnostic method $[2,3]$. Therefore, there is a need for a better understanding of serological tests.

Serological assays are based on the detection of antibodies against microbe-specific antigens. Thus, this technique employs an indirect measure of pathogen recognition that depends on the immunogenicity and specificity of the antigen and on the function of the host immune response. As the humoral immune response and the serological methods used may be influenced by multiple factors, it is important to evaluate variables related to the development of antibody responses and their detection in order to properly interpret serological test results.

We evaluated the effects of combining different numbers of pneumococcal antigens, pre-existing antibody levels, sampling interval, age, and duration of illness on the detection of antibody responses against protein antigens from S. pneumoniae, Haemophilus influenzae, and Moraxella catarrhalis in children with CAP.

\section{Materials and methods}

\section{Study design and participants}

The main group of patients in our investigation was drawn from a clinical trial that compared the efficacy of different posologic schemes of oral amoxicillin to treat nonhospitalized children with CAP (PNEUMOPAC-Efficacy Study) [4]. In brief, that study included 820 children aged 259 months presenting to the Emergency Department of the Federal University of Bahia hospital, Salvador, Northeast Brazil, with respiratory complaints, along with lower respiratory pathology findings and presence of pulmonary infiltrates or consolidation on the chest radiograph taken on admission and read by the pediatrician on duty. Patients with serum samples from the acute and the convalescent phases of the disease available for antibody assay and without previous use of pneumococcal vaccines were selected. Severely malnourished patients - Z-score for weight-for-age under -3.00 [5] — were excluded from the study. "Anthro" software was used for nutritional evaluation.

Another group of patients was evaluated in this investigation. Acute and convalescent serum samples from 13 children with invasive pneumococcal pneumonia were tested in order to help the interpretation of pneumococcal serology results from the main study group. These patients with invasive pneumococcal disease were children aged $<5$ years with clinical and radiological diagnosis of pneumonia hospitalized in the Federal University of Bahia hospital. Invasive disease was diagnosed by blood culture $(n=9)$ or polymerase chain reaction (PCR) for Ply $(n=4)$. All of these children had not received any pneumococcal vaccine. Data about this group have been previously published [6].

Acute serum samples were collected upon hospital presentation and convalescent serum samples were collected on the follow-up visit. Duration of illness was calculated by the duration of fever or respiratory symptoms, whichever was the longest, reported by legal guardians upon hospital presentation.

All legal guardians provided written informed consent before enrolment in the investigation. The study was approved by the Ethics Committee of the Federal University of Bahia and it was conducted in accordance with the principles of the Declaration of Helsinki.

\section{Procedures}

The description of the serology protocol employed and the procedures for serum sample analyses have been previously published [7]. Briefly, acute and convalescent serum samples were assayed with a multiplexed bead-based serological test, using Luminex $\mathrm{xMAP}^{\circledR}$ Technology, for the investigation of IgG antibodies against eight recombinant pneumococcal protein antigens (pneumolysin [Ply], choline binding protein A [CbpA], pneumococcal surface protein A families 1 and 2 [PspA 1 and PspA 2], pneumococcal choline binding protein A [PcpA], pneumococcal histidine triad protein D [PhtD], serine/threonine protein kinase [StkP-C, SP1732-3], and protein required for cell wall separation of group B streptococcus [PcsB-N, SP2216-1]), three H. influenzae recombinant protein antigens (NTHi Protein D, NTHi0371-1, and NTHi0830), and five $M$. catarrhalis recombinant protein antigens (outer membrane protein CD, MC_RH4_2506, MC_RH4_1701, MC_RH4_3729-1, and MC_RH4_4730). Nine bead sets were used to couple with the antigens: Ply, CbpA, PcpA, PhtD, StkP-C, and PcsB-N were conjugated in one bead region each; PspA 1 and PspA 2 were conjugated in the same bead region; due to resource restrictions, all $H$. influenzae and all M. catarrhalis proteins were conjugated onto one bead region per bacterium.

The mean fluorescence intensity (MFI) values provided an indirect measure of the antibody concentrations. The MFI value of each sample was an average of true duplicates. Acute and convalescent serum samples were assayed on the same plate, and positive and negative controls were analyzed on each plate. Samples were assayed using 1:400 and 1:1,600 dilutions, as these dilutions were in the linear range of the 
dilution curve, especially the dilution of 1:1,600, and further dilutions were performed if needed [7]. Herein, the results of antibody levels of acute serum samples are reported for the dilution of 1:1,600. Positive antibody responses were determined by the detection of $\geq 2$-fold rises in antibody levels between the acute and convalescent serum samples. A positive antibody response against $S$. pneumoniae was defined as a positive antibody response against at least one pneumococcal antigen.

\section{Statistical analysis}

Categorical variables are presented as absolute number (percentage) and continuous variables as median (interquartile range [IQR]), as all continuous variables showed a nonparametric distribution. We determined the rate of antibody responses against $S$. pneumoniae for every possible combination of the studied pneumococcal proteins. The influence of different variables on the detection of antibody responses was assessed using multiple logistic regression with robust variance. When deemed appropriate, the optimal cut-off points of certain variables that most reliably discriminated detection or no detection of antibody responses were calculated using receiver operating characteristic (ROC) curves. The sensitivity of these ROC curves expresses the proportion of patients with detection of antibody responses and high values for the studied variable, while the specificity expresses the proportion of patients without detection of antibody responses and low

Table 1 Median of the mean fluorescence intensity (MFI) values of antibodies against each investigated antigen in acute-phase sera (using a 1:1,600 dilution of the samples) from non-hospitalized children with pneumonia

\begin{tabular}{|c|c|c|}
\hline \multirow[t]{2}{*}{ Antigen } & \multicolumn{2}{|c|}{ Antibody level in acute sera } \\
\hline & Median $\left(\mathrm{IQR}^{\mathrm{a}}\right)$ & Range \\
\hline \multicolumn{3}{|l|}{ Streptococcus pneumoniae } \\
\hline CbpA & $4,181.5(866.8-9,756.3)$ & $25-24,441$ \\
\hline PcsB-N & $1,851(403.5-4,909)$ & $21-18,343$ \\
\hline $\mathrm{PhtD}$ & $1,675.5(439.3-3,703)$ & $32-21,044$ \\
\hline PcpA & $819.5(213.3-1,836.8)$ & $23-8,132$ \\
\hline $\mathrm{PspA}^{\mathrm{b}}$ & $300(103-848.5)$ & $34-11,692$ \\
\hline StkP-C & $291.5(89-786.5)$ & $21-6,510$ \\
\hline Ply & $125(63.8-252.3)$ & $27-3,471$ \\
\hline Haemophilus influenzae ${ }^{\mathrm{c}}$ & $140(89-245.3)$ & $32-1,347$ \\
\hline Moraxella catarrhalis ${ }^{\mathrm{d}}$ & $110(76.8-168)$ & $37-1,883$ \\
\hline
\end{tabular}

${ }^{\text {a }}$ Interquartile range

${ }^{\mathrm{b}}$ PspA 1 and PspA 2 were conjugated onto the same bead region

${ }^{\mathrm{c}}$ Three H. influenzae antigens were investigated: NTHi Protein D, NTHi0371-1, and NTHi0830

${ }^{\mathrm{d}}$ Five $M$. catarrhalis antigens were investigated: $\mathrm{MC}$ Omp CD, $\mathrm{MC}$ RH4_2506, MC_RH4_1701, MC_RH4_3729-1, and MC_RH4_4730

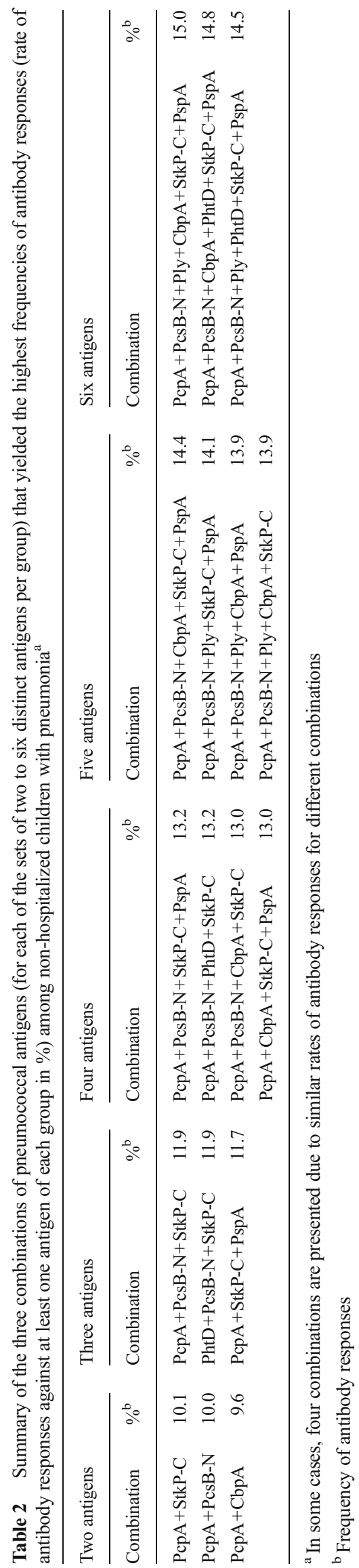


Table 3 Median values (interquartile range) of antibody levels of acute serum samples, sampling interval, age, and duration of illness of nonhospitalized children with pneumonia with and without antibody responses against each investigated antigen

\begin{tabular}{|c|c|c|c|c|c|}
\hline Antigen & Antibody response & $\begin{array}{l}\text { Antibody levels of acute } \\
\text { serum samples (MFI) }\end{array}$ & Sampling interval (days) & Age (months) & $\begin{array}{l}\text { Duration of } \\
\text { illness (days) }\end{array}$ \\
\hline \multicolumn{6}{|l|}{ Streptococcus pneumoniae } \\
\hline \multirow[t]{2}{*}{ Ply } & Yes $(n=24)$ & $90.5(44.3-139.8)$ & $19.5(16-22.3)$ & $16.6(13.4-37)$ & $7(4-8)$ \\
\hline & No $(n=666)$ & $129.5(66-256)$ & $19(15-21)$ & $26.9(14.6-41.2)$ & $5(4-8)$ \\
\hline \multirow[t]{2}{*}{ CbpA } & Yes $(n=35)$ & $901(88-2,018)$ & $19(16-21)$ & $27.1(14.4-39.1)$ & $5(4-8)$ \\
\hline & No $(n=655)$ & 4,801 (964-9,902) & $19(15-21)$ & $26.7(14.4-41.4)$ & $6(4-8)$ \\
\hline \multirow[t]{2}{*}{ PspA } & Yes $(n=29)$ & $191(65.5-413)$ & $17(15-22)$ & $29.7(15.5-40.5)$ & $5(4-7)$ \\
\hline & No $(n=661)$ & $317(107-899)$ & $19(15-21)$ & $26.6(14.2-41.1)$ & $6(4-9)$ \\
\hline \multirow[t]{2}{*}{ PcpA } & Yes $(n=49)$ & $207(47.5-733)$ & $19(16.5-21)$ & $20.9(12-35.4)$ & $6(4-8)$ \\
\hline & No $(n=641)$ & $915(278-1,888)$ & $19(15-21)$ & $26.9(14.7-41.6)$ & $5(4-8)$ \\
\hline \multirow[t]{2}{*}{$\mathrm{PhtD}$} & Yes $(n=31)$ & $694(74-2,335)$ & $20(17-21)$ & $26.4(16.1-39.6)$ & $7(5-8)$ \\
\hline & No $(n=659)$ & $1,727(476-3,928)$ & $19(15-21)$ & $26.7(14.3-41.1)$ & $5(4-8)$ \\
\hline \multirow[t]{2}{*}{ StkP-C } & Yes $(n=32)$ & $72.5(31.3-339.5)$ & $18.5(16-21)$ & $20.1(14.3-35.2)$ & $7(4-8)$ \\
\hline & No $(n=658)$ & $301(97-803.5)$ & $19(15-21)$ & $26.9(14.5-41.3)$ & $5(4-8)$ \\
\hline \multirow[t]{2}{*}{ PcsB-N } & Yes $(n=47)$ & $666(117-1,434)$ & $18(16-21)$ & $26.4(13.5-34.8)$ & $7(4-10)$ \\
\hline & No $(n=643)$ & $2,014(451-5,047)$ & $19(15-21)$ & $26.7(14.6-41.4)$ & $5(4-8)$ \\
\hline \multirow[t]{2}{*}{ Haemophilus influenzae } & Yes $(n=40)$ & $89(57.3-165)$ & $19.5(17-22)$ & $15.9(10-35.5)$ & $7(4-11)$ \\
\hline & No $(n=650)$ & $143(91-250)$ & $19(15-21)$ & $27.2(14.8-41.4)$ & $5(4-8)$ \\
\hline \multirow[t]{2}{*}{ Moraxella catarrhalis } & Yes $(n=16)$ & $147.5(65.8-281.3)$ & $19.5(15-25.5)$ & $16.6(12.1-33.3)$ & $5(5-8)$ \\
\hline & No $(n=674)$ & $110(77-167)$ & $19(15-21)$ & $26.8(14.5-41.1)$ & $6(4-8)$ \\
\hline
\end{tabular}

values for the studied variable. Statistical tests were twotailed, with a significance level of 0.05 . The software Stata (version 13.0) was used for the analyses.

\section{Results}

Out of the 820 patients from the PNEUMOPAC-Efficacy Study, exclusions included the following: 81 (9.9\%) did not have available paired serum samples, 45 (5.5\%) had received at least one dose of pneumococcal vaccines, three $(0.4 \%)$ did not have the vaccine card checked, and one $(0.1 \%)$ was severely malnourished. Therefore, our study group of nonhospitalized patients comprised the remaining 690 children. The median age was 26.7 (IQR: 14.5-41) months and there were $351(50.9 \%)$ males. The median time interval between serum sample collection was 19 (IQR: 15-21) days. The median duration of illness was 5 (IQR: 4-8) days. The most frequent symptoms were cough $(97.8 \%$; median [IQR] duration of 6 [3-9] days), fever (93\%; median [IQR] duration of 4 [2-5] days), and difficulty breathing (62\%; median [IQR] duration of 3 [2-5] days). The median MFI values of antibodies against each investigated antigen in the acute sera are shown in Table 1.

The frequency of positive antibody responses was $15.4 \%$ $(n=106)$ for S. pneumoniae, $5.8 \%(n=40)$ for $H$. influenzae, and $2.3 \%(n=16)$ for M. catarrhalis. Overall, the rate of simultaneous antibody responses was $1.6 \%(n=11)$ for
$S$. pneumoniae and $H$. influenzae, $0.6 \%(n=4)$ for S. pneumoniae and M. catarrhalis, and $0.4 \%(n=3)$ for S. pneumoniae, H. influenzae and M. catarrhalis. The detection rate of at least one of the three bacteria was $20.4 \%(n=141)$.

Overall, the rate of antibody response against each pneumococcal antigen was $7.1 \%$ for PcpA, $6.8 \%$ for PcsB-N, $5.1 \%$ for CbpA, $4.6 \%$ for StkP-C, $4.5 \%$ for PhtD, $4.2 \%$ for PspA, and $3.5 \%$ for Ply. The seven pneumococcal antigens (PspA 1 and PspA 2 were considered as one) were grouped in every possible combination: 21 groups of two antigens, 35 groups of three antigens, 35 groups of four antigens, 21 groups of five antigens, and seven groups of six antigens. Among these different combinations, the frequencies of antibody responses against at least one pneumococcal protein varied from 6.1 to $10.1 \%$ for groups of two antigens, from 8.1 to $11.9 \%$ for groups of three antigens, from 9.9 to $13.2 \%$ for groups of four antigens, from 11.9 to $14.4 \%$ for groups of five antigens, and from 13.5 to $15 \%$ for groups of six antigens. Table 2 shows the combinations of pneumococcal antigens, arranged in groups from two to six, that yielded the highest frequencies of antibody responses.

The median values of antibody levels of acute serum samples, sampling interval, age, and duration of illness among patients with and without antibody response to each investigated antigen are shown in Table 3. The results of the multiple logistic regression analysis evaluating the effects of the aforementioned factors on antibody responses against protein antigens from the three studied bacteria are shown in Table 4 . The 
levels of antibodies against the investigated pneumococcal (except PspA and StkP-C) and H. influenzae proteins in acute serum samples were negatively associated with the detection of antibody responses against these antigens. The sampling interval was positively associated with the detection of antibody responses against Ply, CbpA, and H. influenzae proteins. Duration of illness was negatively associated with antibody responses against PspA. There was no association between the age of the patients and the detection of antibody responses.

The ROC curves for the determination of the optimal cutoff points of sampling interval that most reliably discriminated detection or no detection of antibody responses are presented in Fig. 1. The best cut-off point for the sampling interval was 21 days (sensitivity: $45.8 \%$; specificity: $67.9 \%$ ) for Ply, 19 days (sensitivity: $54.3 \%$; specificity: $48.4 \%$ ) for CbpA, and 21 days (sensitivity: $47.5 \%$; specificity: $68.3 \%$ ) for H. influenzae antigens. The optimal time interval could be summarized as being equal to 3 weeks for the detection of antibody responses against $\mathrm{Ply}, \mathrm{CbpA}$, and $H$. influenzae proteins.

Regarding the group of children with invasive pneumococcal disease, there were serum samples available from $13 \mathrm{pa}-$ tients. The median age of these children was 13.9 (IQR: 9.9$24.8)$ months, and there were $10(76.9 \%)$ males. The frequency of antibody responses against pneumococcal protein antigens in this group was $92.3 \%(n=12)$.

\section{Discussion}

Our results demonstrated that the combination of different pneumococcal proteins in serological assays increases the rate of detection of antibody responses in non-hospitalized children with CAP. We also found that pre-existing antibody levels and sampling interval affect the detection of antibody responses against protein antigens from $S$. pneumoniae and $H$. influenzae in children aged $<5$ years with CAP. Furthermore, we present cut-off points of the sampling interval for the detection of antibody responses against some of the investigated antigens.

Few studies have investigated antibody responses against protein antigens from S. pneumoniae, except for Ply [8-10], in children with CAP in this age group. Posfay-Barbe et al. measured IgG antibodies against five pneumococcal proteins (Ply, PhtD, PcpA, PhtE, and LytB) in paired serum samples of children aged $\leq 6$ years hospitalized with CAP [11]. The rates of $\geq 2$-fold increases in antibody levels in that study were $21 \%, 27 \%$, and $31 \%$ for Ply, PhtD, and PcpA, respectively. The higher rates of antibody responses against Ply, PhtD, and PcpA found by Posfay-Barbe et al. when compared to our study might be due to differences between the study populations. For instance, the former study only enrolled hospitalized patients with CAP, while our main study group comprised 


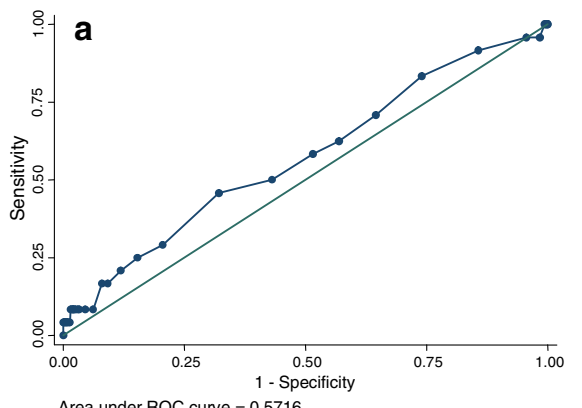

Area under ROC curve $=0.5716$

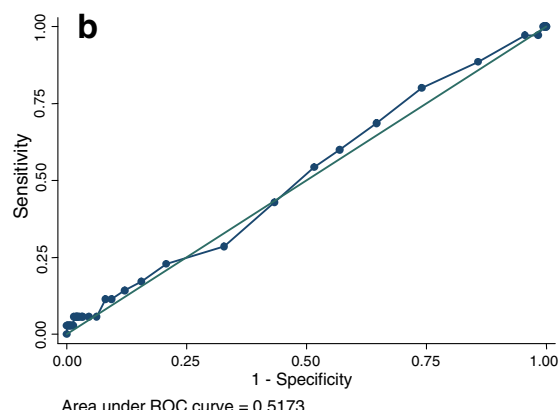

Area under ROC curve $=0.5173$

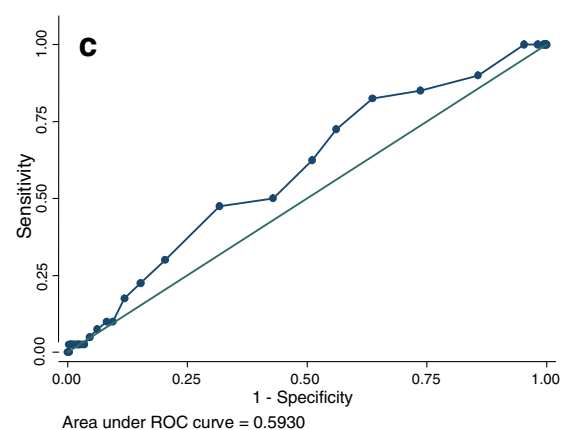

Area under ROC curve $=0.5930$

Fig. 1 Receiver operating characteristic (ROC) curve for the association of antibody responses against Ply (a), CbpA (b), and Haemophilus influenzae protein antigens (c) with sampling interval for non-hospitalized children with pneumonia

patients with non-severe CAP treated as outpatients. It has been previously reported that children with CAP treated as inpatients are more frequently diagnosed with a pneumococcal infection $[8,12]$. To the best of our knowledge, no serological tests using recombinant protein antigens from H. influenzae or M. catarrhalis among children with CAP have been published to date.

The use of combinations of different pneumococcal proteins demonstrated an increase in the rate of positive antibody responses against $S$. pneumoniae and the highest sensitivity for antibody response detection was achieved when we combined all antigens. For instance, the combination of seven antigens detected over $10 \%$ more cases of antibody responses in comparison to the rate of detection of responses against most pneumococcal antigens separately. Notably, herein, PcpA and PcsB$\mathrm{N}$ regularly figured among the combinations of pneumococcal antigens that resulted in the highest rates of antibody responses against $S$. pneumoniae (Table 2). These results are in accordance with the findings from Posfay-Barbe et al., who reported that the use of combinations of pneumococcal protein antigens may markedly improve the sensitivity of antibody response detection against S. pneumoniae, particularly if PcpA was included in the combination [11]. The use of combinations with fewer antigens and higher rates of antibody responses could potentially decrease the cost of serological assays.

Our study showed that pre-existing antibody levels are negatively associated with the detection of antibody responses against proteins from $S$. pneumoniae (for all antigens investigated, except PspA and StkP-C) and H. influenzae. Posfay-Barbe et al. found similar results for pre-existing antibodies levels against PcpA and the detection of S. pneumoniae by different diagnostic methods (blood culture, Ply PCR, and serology using the protein antigens PcpA, Ply, PhtD, PhtE, or LytB) in children with CAP [11]. It has also been demonstrated that pre-existing antibody levels are negatively associated with the detection of antibody responses against pneumococcal polysaccharides after unconjugated pneumococcal vaccine exposure [13]. One possible explanation for the influence of preexisting antibody levels on the detection of antibody responses in these studies is the occurrence of a ceiling effect of antibody production. That is, when pre-existing antibody levels are already high, it may be difficult to raise the levels any further. An alternative explanation would be that high antibody levels in acute serum samples reflect a certain level of immune protection against these bacteria, probably acquired in previous exposures to their antigens. To our knowledge, no previous studies evaluated such associations for antibodies against H. influenzae and M. catarrhalis proteins in children with CAP.

A longer sampling interval was associated with higher rates of antibody responses against two pneumococcal proteins (Ply and $\mathrm{CbpA}$ ) and $H$. influenzae proteins. It was demonstrated that a sampling interval of 3 weeks is an optimal cut-off point for the detection of antibody responses against Ply, CbpA, and H. influenzae proteins. Thus, studies investigating antibody responses against these antigens in children with CAP could benefit from scheduling the sampling interval to be 3 weeks. Shorter duration of illness was associated with higher rates of antibody responses against PspA. This might be explained by a relatively fast and brief production of antibodies against this antigen upon pathogen exposure in childhood CAP. Age was not shown to influence the detection of antibody responses against protein antigens from the investigated bacteria.

The limitations of our study need to be recognized. First, the absence of a threshold fold increase in antibody levels associated with acute infection by each of the three investigated bacteria might undermine the interpretation of analyses involving antibody responses. However, we found that $\geq 2$-fold rises in antibody levels detected $92.3 \%$ of the cases of invasive pneumococcal pneumonia. Thus, this threshold fold increase might be suitable for the detection of antibody responses in children with pneumococcal pneumonia using protein antigens. Another limitation is that we do not have data from our study group about previous infections caused by the investigated bacteria. Patients undergoing recurrence of infection by one of these agents might demonstrate different patterns of antibody responses compared to patients undergoing primary infection. Additionally, in order to provide further details regarding serology using $H$. influenzae and $M$. catarrhalis proteins, we suggest that future studies evaluate the role of each protein antigen from these pathogens individually. 
In conclusion, we found that a combination of pneumococcal protein antigens in serological assays, especially combinations including PcpA and PcsB-N, enhances the rate of detection of antibody responses against $S$. pneumoniae in nonhospitalized children aged $<5$ years with CAP. In addition, we showed that pre-existing antibody levels are negatively associated with the detection of antibody responses against $S$. pneumoniae and $H$. influenzae proteins in children with CAP. Furthermore, we demonstrated that 3 weeks might be the optimal sampling interval for the detection of antibody responses against some pneumococcal and $H$. influenzae proteins in children with CAP. Duration of illness was negatively associated with antibody responses against PspA and patient age did not affect the detection of antibody responses against the investigated antigens. Antibody responses against $M$. catarrhalis proteins were not shown to be influenced by the studied factors.

Acknowledgments We thank Sanofi Pasteur (Lyon, France) for supplying PcpA and PhtD; Prof. Elaine Tuomanen at St. Jude Children's Research Hospital (Memphis, TN, USA) for supplying Ply, CbpA, PspA 1; Profs. Susan Hollingshead, David Briles, and Pat Coan at University of Alabama (Birmingham, AL, USA) for supplying PspA 2; and Valneva Austria GmbH (Vienna, Austria) for supplying SP1732-3, SP2216-1, NTHi Protein D, NTHi0371-1, NTHi0830, MC Omp CD, MC_RH4 2506, MC_RH4_1701, MC_RH4_3729-1, and MC_RH4_4730. We also thank Camilla Virta and Leena Saarinen for their help in the laboratory and thank Ângela G. Vasconcellos, MD, PhD student, for her technical assistance with the ROC curve analysis.

This work was supported by: Bahia State Agency for Research Funding (FAPESB), Brazil; Brazilian Council for Scientific and Technological Development (CNPq), Brazil; and, in part, by National Institute for Health and Welfare, Finland. Profs. Aldina Barral, Maria Regina A. Cardoso, and Cristiana M. Nascimento-Carvalho are investigators at CNPq.

Conflict of interest Andreas Meinke is an employee of Valneva Austria $\mathrm{GmbH}$.

\section{References}

1. Walker CL, Rudan I, Liu L, Nair H, Theodoratou E, Bhutta ZA et al (2013) Global burden of childhood pneumonia and diarrhoea. Lancet 381:1405-1416
2. Murdoch DR, O'Brien KL, Driscoll AJ, Karron RA, Bhat N; Pneumonia Methods Working Group; PERCH Core Team (2012) Laboratory methods for determining pneumonia etiology in children. Clin Infect Dis 54:S146-S152

3. Korppi M, Leinonen M, Ruuskanen O (2008) Pneumococcal serology in children's respiratory infections. Eur J Clin Microbiol Infect Dis 27:167-175

4. Vilas-Boas A-L, Fontoura M-S, Xavier-Souza G, Araújo-Neto CA, Andrade SC, Brim RV et al (2014) Comparison of oral amoxicillin given thrice or twice daily to children between 2 and 59 months old with non-severe pneumonia: a randomized controlled trial. J Antimicrob Chemother 69:1954-1959

5. World Health Organization (WHO) (2008) Training course on child growth assessment. WHO child growth standards. WHO, Geneva, Switzerland. Available online at: http://whqlibdoc.who.int/ publications/2008/9789241595070 A eng.pdf. Accessed 13 July 2009

6. Nascimento-Carvalho CM, Cardoso MR, Barral A, Araújo-Neto CA, Guerin S, Saukkoriipi A et al (2010) Procalcitonin is useful in identifying bacteraemia among children with pneumonia. Scand J Infect Dis 42:644-649

7. Andrade DC, Borges IC, Laitinen H, Ekström N, Adrian PV, Meinke A et al (2014) A fluorescent multiplexed bead-based immunoassay (FMIA) for quantitation of $\mathrm{IgG}$ against Streptococcus pneumoniae, Haemophilus influenzae and Moraxella catarrhalis protein antigens. J Immunol Methods 405:130-143

8. Heiskanen-Kosma T, Korppi M, Jokinen C, Kurki S, Heiskanen L, Juvonen H et al (1998) Etiology of childhood pneumonia: serologic results of a prospective, population-based study. Pediatr Infect Dis J 17:986-991

9. Juvén T, Mertsola J, Toikka P, Virkki R, Leinonen M, Ruuskanen O (2001) Clinical profile of serologically diagnosed pneumococcal pneumonia. Pediatr Infect Dis J 20:1028-1033

10. Cevey-Macherel M, Galetto-Lacour A, Gervaix A, Siegrist CA, Bille J, Bescher-Ninet B et al (2009) Etiology of communityacquired pneumonia in hospitalized children based on WHO clinical guidelines. Eur J Pediatr 168:1429-1436

11. Posfay-Barbe KM, Galetto-Lacour A, Grillet S, Ochs MM, Brookes RH, Kraehenbuhl JD et al (2011) Immunity to pneumococcal surface proteins in children with community-acquired pneumonia: a distinct pattern of responses to pneumococcal choline-binding protein A. Clin Microbiol Infect 17:1232-1238

12. Nascimento-Carvalho CM (2001) Etiology of childhood community acquired pneumonia and its implications for vaccination. Braz $\mathrm{J}$ Infect Dis 5:87-97

13. Hare ND, Smith BJ, Ballas ZK (2009) Antibody response to pneumococcal vaccination as a function of preimmunization titer. $\mathrm{J}$ Allergy Clin Immunol 123:195-200 